\title{
SEMIDIRECT PRODUCTS OF TOPOLOGICAL GROUPS WITH EQUAL UNIFORMITIES
}

\author{
R. W. BAGLEY AND K. K. LAU
}

Abstract. If $H$ (S) $K$ is a semidirect product such that the automorphisms of $K$ induced by $H$ are inner automorphisms, $H$ has equal uniformities and $K$ is compact, then $H$ (S) $K$ has equal uniformities. Other closely related results are obtained and used to give an alternate proof that a semidirect product $V(S)$ of a vector group and a compact group is isomorphic to the direct product $V \times K$.

Let $H$ and $K$ be topological groups and let $\phi: H \times K \rightarrow K$ be a continuous mapping such that each mapping $\tau_{h}: K \rightarrow K$, defined by $\tau_{h}(k)=\phi(h, k)$, is an automorphism of $K$ and the mapping $h \rightarrow \tau_{h}$ is a homomorphism of $H$ into the group of automorphisms $A(K)$ of $K$. By $H$ (S) $K$ we mean the (topological) semidirect product with group operation, $(h, k)(g, l)=\left(h g, \tau_{g}(k) l\right)$, and product topology of $H \times K$.

We say that a topological group has equal uniformities if its left uniformity and right uniformity are equal. Even in quite restrictive cases semidirect products of groups with equal uniformities may fail to have equal uniformities. We obtain sufficient conditions for a semidirect product to have equal uniformities and show how such a result can be applied to obtain a structure theorem. We were led to our results by the work in [4]. Many of the results of [4] appear in [5] and [6].

If $(G, \sigma)$ is a topological group with topology $\sigma$, then there is a minimum topology $\rho \supset \sigma$ such that $(G, \rho)=G^{*}$ is a topological group with equal uniformities. If $v$ is a neighborhood base at the identity of $(G, \sigma)$, then $\left\{\cap_{t \in G} t V t^{-1}: V \in V\right\}$ is a neighborhood base at the identity of $G^{*}$. We let $V^{*}=\bigcap_{t \in G} t V t^{-1}$.

By $\Pi_{K}$ we mean the mappings $\left\{\Pi_{k}: H \rightarrow K: k \in K\right\}$ where $\Pi_{k}(h)$ $=\tau_{h}(k)$. We say that $H$ (s) $K$ is a strong semidirect product if the corresponding homomorphism $\phi$ carries $H$ into $I(K)$, the group of inner automorphisms of $K$.

Lemma 1. If $H$ and $K$ have equal uniformities and $\Pi_{K}$ is equicontinuous, then the strong semidirect product $H$ (s) $K$ has equal uniformities.

Received by the editors October 13, 1969.

AMS 1969 subject classifications. Primary 2210; Secondary 2052.

Key words and phrases. Semidirect products of topological groups, equal uniformities, compact groups. 
Proof. Let $U$ and $V$ be invariant neighborhoods (invariant under inner automorphisms) of the identities of $H$ and $K$ respectively. In particular $\tau_{h}(V)=V$ for each $h$. Then

$$
\begin{aligned}
(U \times V)^{*} & =\bigcap_{h, k}\left(e, \tau_{h^{-1}}(k)\right)(h, e) U \times V\left(h^{-1}, e\right)\left(e, \tau_{h^{-1}}\left(k^{-1}\right)\right) \\
& =\bigcap_{h, k}\left(e, \tau_{h^{-1}}(k)\right) U \times V\left(e, \tau_{h^{-1}}\left(k^{-1}\right)\right)=\bigcap_{k}(e, k) U \times V\left(e, k^{-1}\right) .
\end{aligned}
$$

Let $W$ be an invariant neighborhood of the identity of $K$ such that $W^{2} \subset V$ and let $U_{1}$ be a neighborhood of the identity of $H$ such that $U_{1} \subset U$ and $\tau_{u}(k) \in W k$ for all $u \in U_{1}$ and $k \in K$. If $(u, w) \in U_{1} \times W$, then $(e, k)(u, w)\left(e, k^{-1}\right)=\left(u, \tau_{u}(k) w k^{-1}\right)=\left(u, \tau_{u}(k) k^{-1} k w k^{-1}\right) \in U$ $\times W^{2} \subset U \times V$. Thus $(U \times V)^{*}$ is a neighborhood of the identity in $H$ (S $K$ as desired.

By $G \cong H$ we mean $G$ and $H$ are topologically isomorphic.

THEOREM 1. If $H$ (s) $K$ is a strong semidirect product of the topological groups $H$ and $K$ such that $\Pi_{K}$ is equicontinuous on $H^{*}$ into $K^{*}$, then $(H \text { (s) } K)^{*} \cong H^{*}$ (s) $K^{*}$.

Proof. To show that $H^{*}$ (s) $K^{*}$ is well defined it is sufficient to show that $\phi: H^{*} \times K^{*} \rightarrow K^{*}$ is continuous. Let $h_{0}, k_{0}$ be arbitrary elements of $H, K$ and let $W_{0}$ be a neighborhood of the identity of $K$. Choose another neighborhood $W$ such that $W^{2} \subset W_{0}$ and choose $U$ and $V$ neighborhoods of the identities of $H$ and $K$ such that $\tau_{h_{0}}(V) \subset W$ and $\tau_{z}(k) k^{-1} \in W^{*}$ for each $k \in K$ and $z \in U^{*}$. These neighborhoods can be so chosen by virtue of the continuity of $\tau_{h_{0}}$ and the equicontinuity of $\Pi_{K}$. Now let $x \in h_{0} U^{*}$ and $y \in V^{*} k_{0}$. Then $\tau_{x}(y) \tau_{h_{0}}\left(y^{-1}\right)$ $=\tau_{h_{0}^{-1} x}\left(\tau_{h_{0}}(y)\right) \tau_{h_{0}}\left(y^{-1}\right) \in W^{*} \quad$ since $h_{0}^{-1} x \in U^{*}$ and $\tau_{h_{0}}(y) \tau_{h_{0}}\left(k_{0}^{-1}\right)$ $=\tau_{h_{0}}\left(y k_{0}^{-1}\right) \in W^{*}$ since $y k_{0}^{-1} \in V^{*}$. Therefore $\tau_{x}(y) \tau_{h_{0}}\left(k_{0}^{-1}\right) \in\left(W^{*}\right)^{2}$ $\subset W_{0}^{*}$. It follows that $\phi: H^{*} \times K^{*} \rightarrow K^{*}$ is continuous and, by Lemma $1, H^{*}$ (s) $K^{*}$ has equal uniformities. To complete the proof of the theorem it is sufficient to show that $(H \odot K)^{*}$ has the product topology $\beta$ of $H^{*} \times K^{*}$.

First, since $(H(S))^{*}$ has the smallest topology on $H \times K$ which makes $H$ (5) $K$ a topological group and which contains the product topology on $H \times K$, it follows that the topology of $(H(K) *$ is contained in $P$. To prove the reverse inclusion we show that the projections of $(H(5))^{*}$ into $H^{*}$ and $K^{*}$ are continuous. For $H^{*}$ the proof is trivial. To prove that the projection of $(H \odot K)^{*}$ into $K^{*}$ is continuous let $W$ be a neighborhood of the identity of $K$. Choose a neighborhood $V$ of the identity of $K$ such that $V^{2} C W$ and choose a neighborhood $U$ of the identity of $H$ such that $z \in U^{*}$ implies that 
$\tau_{z}(k) \in k^{-1} V$ for all $k \in K$. Let $\left(h_{0}, k_{0}\right) \in H \times K$ and let $(x, y) \in\left(h_{0}, e\right)$ $\cdot(U \times V)^{*}\left(e, k_{0}\right)$ which is a neighborhood of $\left(h_{0}, k_{0}\right)$ in $(H \text { (5) } K)^{*}$. Then $\left(h_{0}^{-1} x, y k_{0}^{-1}\right) \in(U \times V)^{*}$ and $(h, k)\left(h_{0}^{-1} x, y k_{0}^{-1}\right)\left(h^{-1}, \tau_{h}^{-1}\left(k^{-1}\right)\right) \in U \times V$

for all $h, k$. That is,

$$
\left(h h_{0}^{-1} x h^{-1}, \tau_{h}^{-1}\left(\tau_{h_{0}^{-1} x}(k) y k_{0}^{-1}\right) \tau_{h}^{-1}\left(k^{-1}\right)\right) \in U \times V
$$

for all $h, k$. Thus, $h_{0}^{-1} x \in U^{*}$ and $\tau_{h_{0}^{-1} x}(k) y k_{0}^{-1} k^{-1} \in V$ for all $k$, taking $h$ to be $e$ in the latter case. Now $k y k_{0}^{-1} k^{-1}=k \tau_{h_{0}^{-1} x}\left(k^{-1}\right) \tau_{h_{0}^{-1} x}(k) y k_{0}^{-1} k^{-1}$ $\in V^{2} \subset W$. Thus the projection $y$ of $(x, y)$ is in $W^{*} k_{0}$ as desired and the proof is complete.

CoROllaRY 1. If $H$ (s $K$ is a strong semidirect product and $K$ is compact, then $(H \text { (s) } K)^{*} \cong H^{*}$ (s $K$. If, in addition, $H$ has equal uniformities, then $H$ (5) $K$ as equal uniformities.

Proof. Since $K$ is compact $\Pi_{K}$ is equicontinuous. The first statement of the corollary now follows and the second statement is an immediate consequence of the first.

Even if $H$ is discrete cyclic and $K$ is compact there is a semidirect product $H$ (S) $K$ which does not have equal uniformities [1]. The situation is different for strong semidirect products as we see by the following.

CoROllaRY 2. If $H$ (s) $K$ is a strong semidirect product and $H$ is discrete, then $(H \text { (S) } K)^{*} \cong H$ (5 $K^{*}$. If, in addition, $K$ has equal uniformities, then $H$ (5) has equal uniformities.

THEOREM 2. If $H$ (s) $K$ is a semidirect product, $H$ is connected and locally compact and $K$ is compact, then ( $H$ (s $K)^{*} \cong H^{*}$ (s $K$. If, in addition, $H$ has equal uniformities, then $H$ (s) $K$ has equal uniformities.

Proof. By a theorem of Iwasawa (Theorem 1 of [3]) $A(K) / I(K)$ is totally disconnected. Thus, since $H$ is connected, the continuous homomorphism which takes $H$ into $A(K)$ must in fact carry $H$ into $I(K)$. That is, $H$ (S) $K$ is a strong semidirect product. The theorem now follows from Corollary 1 of Theorem 1.

In [1] there is an example of a semidirect product $H$ (5) $K$ where $H$ is discrete and $K$ is compact but $H$ (5) $K$ does not have equal uniformities. Another example of a semidirect product which does not have equal uniformities is the following example given by Ramsay [4].

EXAMPLE. Let $G$ be the semidirect product of $R^{2}$ by the circle group $C$ where the automorphism of $R^{2}$ induced by $e^{i \theta} \in C$ is a rotation 
of $R^{2}$ through the angle $\theta$. $G$ is a locally compact connected group with trivial center. Thus $G$ cannot be isomorphic to the direct product of a vector group and a compact group. It follows that $G$ does not have equal uniformities. See [7, p. 129].

As an application of Theorem 2 we obtain a structure theorem which is Proposition 9.4 of [2]. The proof here makes use of the conclusion on equal uniformities of a semidirect product, whereas in [2] a lemma on maximally almost periodic groups is used.

THEOREM 3. If $V$ (5) $K$ is a semidirect product of a vector group $V$ and a compact group $K$, then $V$ (5) $K \cong V \times K$.

Proof. By Theorem 2, $V$ (5) $K=G$ has equal uniformities. Since $G / G_{0}$ is compact it follows from Corollary XII.1 in [2] that $G$ is maximally almost periodic and the proof of Proposition 9.4 can be copied from the point which $G$ is proven to be maximally almost periodic. An alternative is to note (also by Corollary XII.1) that $G$ is isomorphic to a semidirect product $K^{\prime}$ (s) $V^{\prime}$ of a compact group $K^{\prime}$ and a vector group $V^{\prime}$. Straightforward computation then yields $G \cong V \times K$.

In conclusion we note that, if $H$ and $K$ have equal uniformities and $H$ (S) $K$ is any semidirect product such that $\left\{\tau_{h}: h \in H\right\}$ and $\Pi_{K}$ are equicontinuous, then $H$ (5) $K$ has equal uniformities. The proof of this is essentially the same as that of Lemma 1.

\section{REFERENCES}

1. R. W. Bagley and T. S. Wu, Topological groups with equal left and right uniformities, Proc. Amer. Math. Soc. 18 (1967), 142-147. MR 34 \#7697.

2. K. H. Hofmann and P. Mostert, Splitting in topological groups, Mem. Amer. Math. Soc. No. 43 (1963). MR 27 \#1529.

3. K. Iwasawa, On some types of topological groups, Ann. of Math. (2) 50 (1949), 507-558. MR 10, 679.

4. R. T. Ramsay, Doctoral Dissertation, University of Miami, Coral Gables, Florida, 1967.

5. - On compactification and structure of topological groups, Proc. Amer. Math. Soc. 20 (1969), 585-589. MR 38 \#2238.

6. - Groups with equal uniformities, Canad. J. Math. 21 (1969), 655-659. MR 39 \#7024.

7. A. Weil, $L$ ' intégration dans les groupes topologiques et ses applications, Actualités Sci. Indust., no. 869, Hermann, Paris, 1940. MR 3, 198.

University of Miami, Coral Gables, Florida 33124 